\title{
Reduction of Salivary Gland Damage During Radioiodine Therapy for Differentiated Thyroid Cancers
}

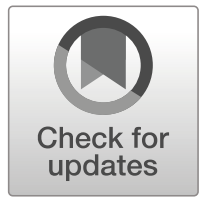

\author{
Byeong-Cheol Ahn ${ }^{1}$ (1)
}

Received: 28 April 2020 / Revised: 5 May 2020 / Accepted: 6 May 2020 / Published online: 12 May 2020

(C) Korean Society of Nuclear Medicine 2020

Radioiodine therapy is one of the major therapeutic modalities in the management of differentiated thyroid cancers (DTCs), and it has been successfully applied in the clinical setting for eight decades. The uptake of radioiodine in DTCs relies on the expression of sodium iodide symporter (NIS), and accumulation of radioiodine induces lethal radiation harm to the lesions through the emission of beta rays $[1,2]$. Salivary glands also express NIS; therefore, they naturally take up radioiodine. Because of the underlying nature of the salivary glands, there is a high prevalence of radioiodine-induced dysfunction followed by damage to the salivary glands. Symptoms of salivary gland dysfunction, including pain, swelling, altered taste, dry mouth, and swallowing/speaking difficulties, are the most serious and common complaints of patients who have undergone radioiodine therapy, and some of these symptoms are lifelong and negatively affect the patient's quality of life [3]. The risk of adverse effect may force strict indications for radioiodine therapy in patients with DTC. Prevention or reduction of radioiodine therapy-induced damage to the salivary glands could increase the active use of effective radioiodine therapy by reducing both patients' and physicians' reluctance to the therapy.

Reducing the accumulation of therapeutic radioiodine in the salivary glands mitigates damage to the glands and can be feasibly assessed on imaging using a gamma camera. Therefore, nuclear medicine technology is one of the most important technologies in researches on salivary gland protection [4]. Although nuclear imaging has not been frequently used to assess radioiodine accumulation in the salivary glands, the application of this technology can provide important

Byeong-Cheol Ahn abc2000@knu.ac.kr

1 Department of Nuclear Medicine, School of Medicine, Kyungpook National University, Kyungpook National University Hospital, 50, Samduk 2-ga, Jung Gu, Daegu 700-721, Republic of Korea information (e.g., kinetic information on radioiodine in the salivary glands) that can assist in the development of strategies for reducing salivary damage [5].

To protect patients from salivary gland damage during radioiodine therapy, various interventions including sialogogues and sufficient water intake have been widely applied. Sialogogues increase salvation and reduce the concentration of radioiodine in the salivary glands, and adequate water intake alleviates dehydration, which decreases salivary lavage [6].

Pilocarpine and amifostine have been used in an attempt to prevent salivary damage; however, the application of these agents is not recommended by various guidelines, mainly because of their limited and debated level of effectiveness as well as their adverse pharmacologic effects [7, 8]. In addition, pharmacologic intervention may reduce the therapeutic effect of radioiodine on DTC lesions.

External massage to the salivary glands has been recommended to expel radioiodine-containing saliva from the glands. Clinical trials of external massage reported reduced accumulation of radionuclides $(\mathrm{Tc}-99 \mathrm{~m}$ or I-123) in the salivary glands and low incidence of salivary gland damage after I-131 therapy [9-11]. Conversely, nonpharmacologic interventions show no systemic effects and do not influence the therapeutic effect of radioiodine on DTC lesions.

Extensive preclinical and clinical research have been conducted on methods to protect the salivary glands during radioiodine therapy. However, prevention of salivary gland damage remains an unmet need in the field of radioiodine therapy, and damage mitigation is desperately warranted to improve the quality of life of patients with DTC who require radioiodine therapy. The development of an optimal protocol to protect the salivary glands during radioiodine therapy may reduce both patients' and physicians' reluctance to use this therapy and broaden the indications for effective radioiodine therapy in patients with DTC. 


\section{Compliance with Ethical Standards}

Conflict of Interest Byeong-Cheol Ahn declares no conflict of interest. There is no source of funding.

Ethical Statement This work does not contain any studies with human participants or animals performed by the author.

\section{References}

1. Hong CM, Ahn BC. Factors associated with dose determination of radioactive iodine therapy for differentiated thyroid cancer. Nucl Med Mol Imaging. 2018;52:247-53.

2. Ahn BC. Sodium iodide symporter for nuclear molecular imaging and gene therapy: from bedside to bench and back. Theranostics. 2012;2:392-402.

3. Jeong SY, Kim HW, Lee SW, Ahn BC, Lee J. Salivary gland function 5 years after radioactive iodine ablation in patients with differentiated thyroid cancer: direct comparison of pre- and postablation scintigraphies and their relation to xerostomia symptoms. Thyroid. 2013;23:609-16.

4. Liu B, Huang R, Kuang A, Zhao Z, Zeng Y, Wang J, et al. Iodine kinetics and dosimetry in the salivary glands during repeated courses of radioiodine therapy for thyroid cancer. Med Phys. 2011;38:5412-9.

5. Jentzen W, Balschuweit D, Schmitz J, Freudenberg L, Eising E, Hilbel T, et al. The influence of saliva flow stimulation on the absorbed radiation dose to the salivary glands during radioiodine therapy of thyroid cancer using ${ }^{124} \mathrm{I}$ PET(/CT) imaging. Eur J Nucl Med Mol Imaging. 2010;37:2298-306.

6. Charalambous A. Seeking optimal management for radioactive iodine therapy-induced adverse effects. Asia Pac J Oncol Nurs. 2017;4:319-22.

7. Haugen BR, Sawka AM, Alexander EK, Bible KC, Caturegli P, Doherty GM, et al. American Thyroid Association Guidelines on the Management of Thyroid Nodules and Differentiated Thyroid Cancer Task Force review and recommendation on the proposed renaming of encapsulated follicular variant papillary thyroid carcinoma without invasion to noninvasive follicular thyroid neoplasm with papillary-like nuclear features. Thyroid. 2017;27:481-3.

8. Francis GL, Waguespack SG, Bauer AJ, Angelos P, Benvenga S, Cerutti JM, et al. Management guidelines for children with thyroid nodules and differentiated thyroid cancer. Thyroid. 2015;25:716-59.

9. Kim HW, Ahn BC, Lee SW, Lee J. Effect of parotid gland massage on parotid gland Tc-99m pertechnetate uptake. Thyroid. 2012;22: 611-6.

10. Hong CM, Son SH, Kim CY, Kim DH, Jeong SY, Lee SW, et al. Emptying effect of massage on parotid gland radioiodine content. Nucl Med Commun. 2014;35:1127-31.

11. Son SH, Lee CH, Jung JH, Kim DH, Hong CM, Jeong JH, et al. The preventive effect of parotid gland massage on salivary gland dysfunction during high-dose radioactive iodine therapy for differentiated thyroid cancer: a randomized clinical trial. Clin Nucl Med. 2019;44:625-33.

Publisher's Note Springer Nature remains neutral with regard to jurisdictional claims in published maps and institutional affiliations. 